\title{
The large-scale environment of groups and clusters of galaxies
}

\author{
Manolis Plionis ${ }^{1,2}$ \\ ${ }^{1}$ Institute of Astronomy \& Astrophysics, National Observatory of Athens, P.Penteli, 15236 \\ Athens, Greece \\ ${ }^{2}$ Instituto Nacional de Astrofísica Óptica y Electrónica, AP 51 y 216, 72000, Puebla, Pue, \\ México
}

\begin{abstract}
It appears that the dynamical status of clusters and groups of galaxies is related to the large-scale structure of the Universe. A few interesting trends have been established:

(1) The Cluster Substructure - Alignment Connection by which clusters show a strong correlation between their tendency to be aligned with their neighbors and their dynamical state (as indicated by the existence of significant substructres).

(2) The Cluster Dynamics -Cluster Clustering Connection by which dynamically young clusters are more clustered than the overall cluster population.

(3) The Cluster-Supercluster Alignment Connection by which clusters of galaxies show a statistical significant tendency to be aligned with the projected major axis orientation of their parent supercluster.

(4) The Galaxy Alignment - Cluster Dynamics Connection by which red-sequence cluster bright galaxies show a significant trend to be aligned with their parent cluster major axis, especially in dynamically young clusters.

(5) The Group Richness - Shape Connection by which groups of galaxies are flatter the poorer they are.

These are strong indications that clusters develop in a hierarchical fashion by anisotropic merging of smaller units along the large-scale filamentary structures within which they are embedded.
\end{abstract}

\section{Introduction}

In the framework of the hierarchical model for the formation of cosmic structures, galaxy clusters are supposed to form by accretion of smaller units (galaxies, groups, etc.). After the epoch of mass aggregation (which depend on the cosmological model), violent relaxation processes will tend to alter the cluster phase-space configuration producing 'regular', quasi-spherical, clusters having a smooth density profile.

Over the last decade, due to the increased spatial resolution in X-ray imaging (RosAT, Xmm-Newton, Chandra) and to the availability of wide-field cameras, many of the previously thought "regular" clusters have shown to be clumpy to some level, a fact that could have important consequences for structure formation theories, since the present fraction of dynamically young clusters, as well as the rate of cluster evolution (as measured for example by their luminosity and temperature functions and their morphology), are cosmology dependent (e.g., Richstone, Loeb \& Turner 1992, Evrard et al. 1993, Lacey \& Cole 1996). Detailed optical and X-ray studies have yielded evidence for significant amounts of subclustering within local and distant clusters (e.g. Buote \& Tsai 1996; Plionis \& Basilakos 2002; Jeltema et al. 2004) indicating that possibly a large fraction is still forming at the present time. Hints do exist for a very recent (within the last Gyr) dynamical evolution of the cluster population (Melott et al. 2001; Plionis 2002, see however 

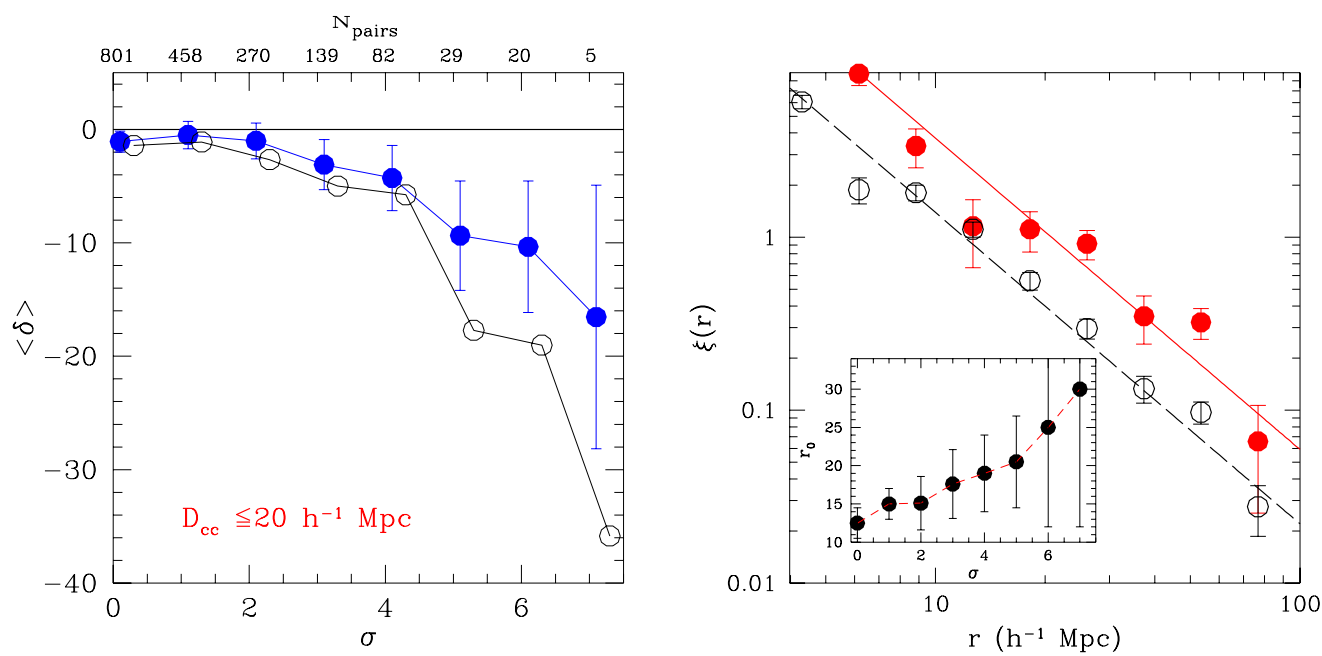

Figure 1. Left PANEL: Alignment signal as a function of substructure significance level. Filled circles correspond to nearest-neighbors and open circles to all neighbors within $20 \mathrm{~h}^{-1} \mathrm{Mpc}$. Right PANEL: The APM cluster correlation function for all the APM clusters (open symbols) and for a sample with significant substructure (filled symbols). The lines represent the best $\left(r / r_{\circ}\right)^{-1.8}$ fit with $r_{\circ} \simeq 12$ and $\simeq 20 h^{-1}$ Mpc respectively. In the insert we show the correlation length as a function of substructure significance level.

Floor et al. 2003). Note however that the existence of substructure does not necessarily mean that the corresponding cluster is dynamically young due to the ambiguity of cluster post-merging relaxation times (see discussion in Plionis 2001).

Furthermore, the dynamical evolution of member galaxies and of the ICM gas is also an open issue. Star formation seems to be active in clusters showing substantial substructure and velocity gradients, as expected if a recent merger has taken place. For example, the fraction of blue galaxies is strongly correlated with cluster ellipticity (Wang \& Ulmer 1997), while ellipticity is strongly correlated with the dynamical state of the cluster (e.g. Kolokotronis et al. 2001). It appears that the violent merging events trigger starformation, possibly through a multitude of different mechanisms; for example, the excess number of galaxy-galaxy interactions, the rapid variation of the cluster gravitational field (Bekki 1999), etc. An interesting observable that may be related to the dynamics of clusters is their tendency to be aligned with their nearest neighbor as well as with other clusters that reside in the same supercluster (e.g. Bingelli 1982; Plionis 1994). Analytical (Bond 1986) and numerical work (e.g. West et al. 1991, van Haarlem \& van de Weygaert 1993, Splinter et al. 1999, Onuora \& Thomas 2000; Faltenbacher et al. 2002; Knebe et al. 2004) have shown that such alignments occur in many hierarchical clustering models and are probably the result of an interesting property of Gaussian random fields which is the "cross-talk" between density fluctuations on different scales (e.g. West 1994).

\section{Environmental trends}

Our aim is to investigate whether there is any relation between the cluster and group large-scale environment and their internal dynamical state. To this end we have analysed APM and Abell clusters (in total more than 1200 clusters) as well as the UzC-SsRs2 (Ramella et al. 2002) and 2DFGRS (Eke et al. 2004) samples of groups of galaxies.

In order to realize our study we need to define, in an objective manner, the dynamical state of the clusters. Evrard et al. (1993) and Mohr et al. (1995) have suggested as 

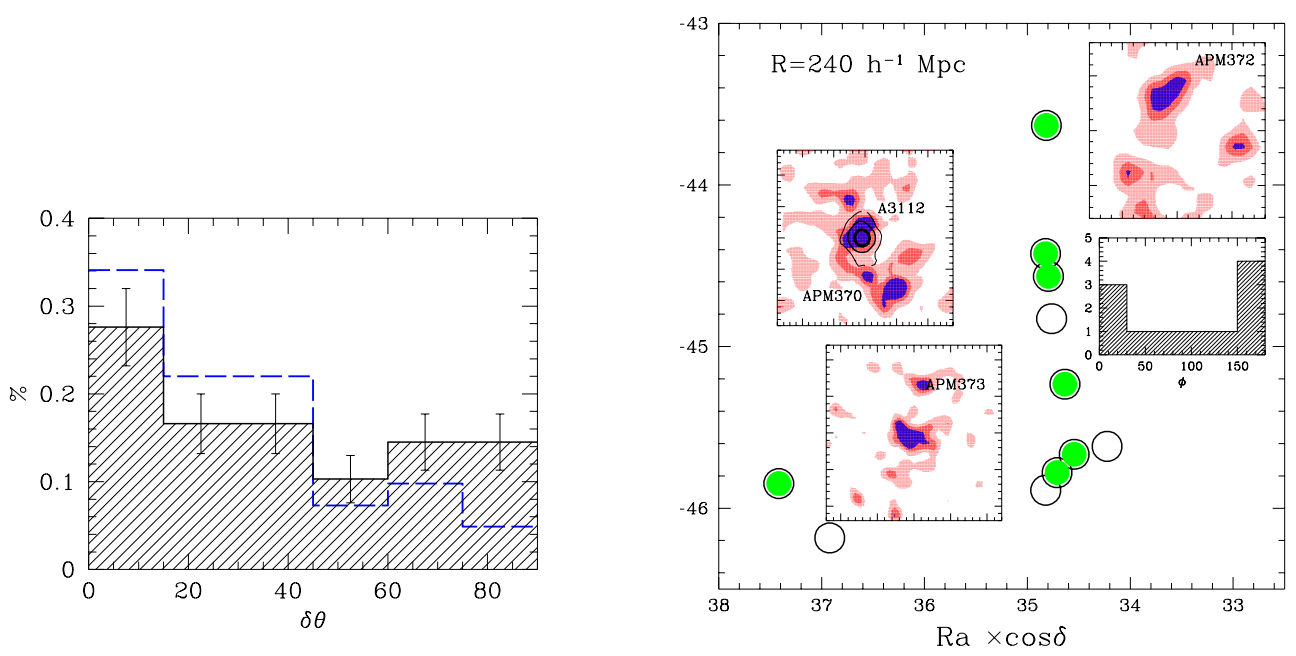

Figure 2. LEFT PANEL: Frequency distribution of the misalignment angle between cluster members and their parent supercluster orientations. The dashed line corresponds to superclusters defined with linking radius of $30 \mathrm{~h}^{-1} \mathrm{Mpc}$ while the hatched distribution to that with linking radius of $20 h^{-1}$ Mpc. Right PANEL: An example of a filamentary APM supercluster containing A3112, A3104, A3111 as well as 9 poorer APM clusters, identified using a linking radius of 12 $h^{-1}$ Mpc. Filled dots represent clusters with significant substructure. In the inserts we show the smooth density distribution for three of the clusters (for the case of A3112 we also show its Rosat-PSPC X-ray contours) as well as the frequency distribution of cluster position angles. The preferred cluster major axis alignment with the projected orientation of the supercluster is evident.

an indicator of cluster substructure the centroid-shift $(s c)$ which is defined as the shift of the cluster center-of-mass position as a function of density threshold above which it is estimated. The significance, $\sigma$, of such centroid variations to the presence of background contamination, random density fluctuations and small galaxy number effects can be estimated using Monte Carlo cluster simulations in which, by construction, there is no substructure (see Plionis 2001). Kolokotronis et al. (2001) calibrated various substructure measures using APM galaxy data and Rosat pointed observations for 22 Abell clusters and found that in most cases using either $\mathrm{X}$-ray or optical data one can identify substructure unambiguously. Their conclusion was that a large and significant centroid-shift is a clear indication of cluster substructure.

Furthermore, in order to investigate the alignment between cluster, group or galaxy orientations, we use the relative position angle between pairs, $\phi_{i, j}$. A significant deviation from the isotropic expectation can be quantified by the measure: $\delta=\sum \phi_{i, j} / N-45$ (Struble \& Peebles 1995), which for an isotropic distribution, has a mean value $\langle\delta\rangle \simeq 0$, and a standard deviation $\sigma=90 / \sqrt{12 N}$. A significantly negative (positive) value of $\delta$ would indicate alignment (misalignment).

Below we present the main results of a variety of studies that indicate a strong correlation between the dynamical state of galaxy structures (groups and clusters) and their large-scale environment.

\subsection{Cluster substructure - Alignment connection}

Plionis \& Basilakos (2002), using a sample of $\sim 900$ APM clusters, correlated the alignment signal between cluster neighbors, $\langle\delta\rangle$, with the existence of cluster substructure, as measured by the deviation from the expected density fluctuations in units of $\sigma$ (e.g. 

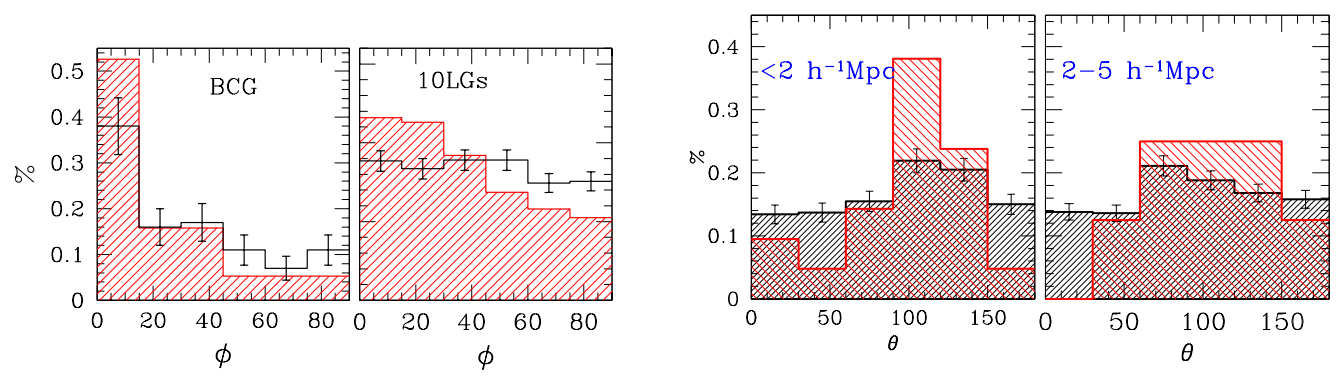

Figure 3. LEFT PANEL: The distribution of misalignment angles $\phi$ between the galaxy and cluster orientations within superclusters for the $v_{\sigma}<900 \mathrm{~km} / \mathrm{sec}$ clusters (black lines) and for those with $v_{\sigma} \geqslant 900 \mathrm{~km} / \mathrm{sec}$ (red hatched histogram). RIGHT PANEL: Histogram of galaxy (black lines) and group (red lines) position angle distribution in Abell 521 for two indicated distance shells.

Kolokotronis et al. 2001; Plionis 2001). In the left panel of Fig. 1 we present $\langle\delta\rangle$ between cluster nearest-neighbors (filled dots) and between all pairs (open dots) with pair separations $<20 h^{-1} \mathrm{Mpc}$. Evidently, there is a strong correlation between the strength of the alignment signal and the substructure significance level. This result confirms statistically the analysis of West et al. (1995) based on EInsteIn X-ray data of $\sim 90$ clusters.

\subsection{Cluster substructure - Clustering connection}

Where do clusters with significant substructure reside? The spatial 2-point cluster correlation function for cluster samples with different substructure significance levels show a strong trend. In the right panel of Fig. 1 we plot the correlation length, $r_{\circ}$, as a function of the cluster substructure significance level, which is clearly an increasing function of $\sigma$. The conclusion of this analysis is that clusters showing evidence of dynamical activity reside in high-density environments. Our results are in agreement with a similar environmental dependence found in the REFLEX and BCS X-ray cluster sample (Schuecker et al., 2001) and for cooling flow clusters with high mass accretion rates (Loken, Melott \& Miller 1999).

\subsection{Cluster-supercluster alignment connection}

A further interesting question regarding environmental effects on large scales is whether clusters are also aligned with the orientation of their parent supercluster. We define APM superclusters by using a friends-of-friends algorithm and estimate for each supercluster the misalignment angle, $\delta \theta$, between its projected orientation and the mean position angle of its member clusters. In Fig. 2 we present the frequency distribution of $\delta \theta$ for two different supercluster catalogues (based on linking radii of 20 and $30 h^{-1} \mathrm{Mpc}$, respectively). The significant excess of small $\delta \theta$ 's is evidence that indeed clusters do show preferential alignments with the orientation of their parent superclusters. As an example, in the right panel of Fig. 2 we present the projected distribution of clusters in a dense and filamentary APM supercluster. Embedded in the plot are the smoothed galaxy density distributions of a few member clusters. It is evident that the cluster position angle distribution reflects the projected orientation of the supercluster.

\subsection{The galaxy alignment - Cluster dynamics connection}

Analysing the DPOSS images of 303 Abell clusters (with $z \leqslant 0.12$ ), Plionis et al. (2003) found significant alignments between the projected major axis of the 10 brightest galaxies (excluding the BCG) and their parent cluster position angle, mostly in high-density 


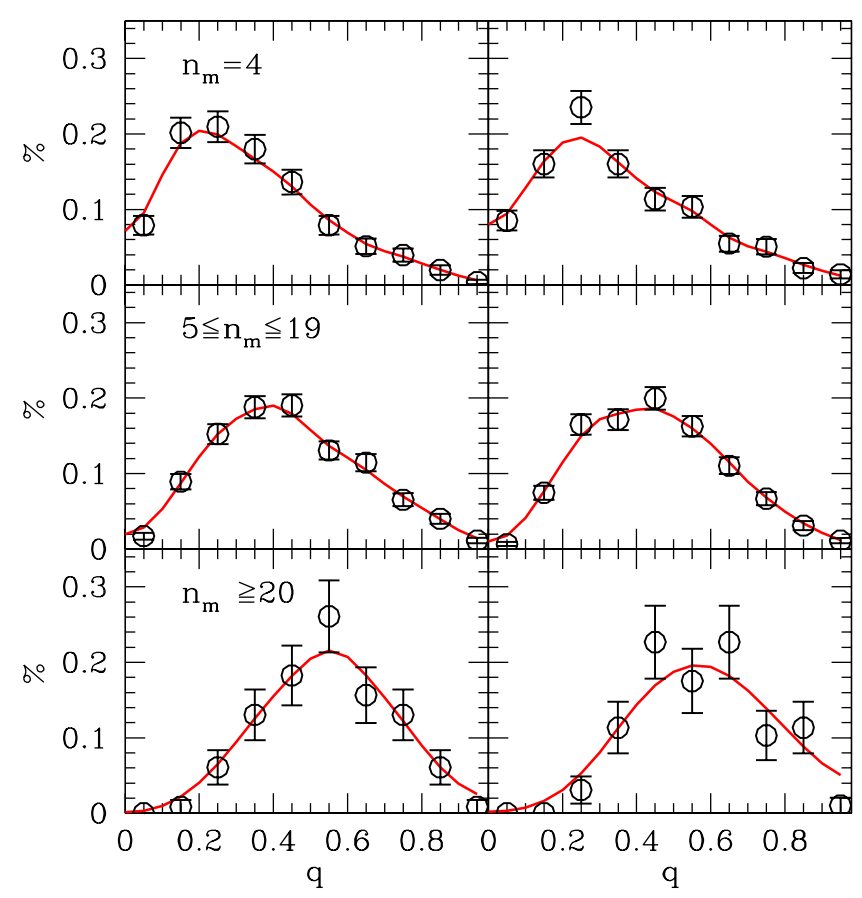

Figure 4. The apparent 2DFGRS group axial ratio distributions for different group membership, $n_{m}$, and for the northern (NGP) and southern (SGP) subsamples respectively The solid line is the smooth fit from the nonparametric kernel estimator.

environments. This alignment signal is stronger for higher velocity dispersion clusters, which in our case (based on a variety of criteria) appear also to be dynamically young merging clusters (see left panel of Fig. 3). This points strongly in the direction of galaxy alignments being correlated with their parent cluster dynamical youth and local density (see also West \& Blakeslee 2000; Knebe et al. 2004). We have also studied in detail, using very deep WFI images, the highly unrelaxed Abell cluster (A521 at $z=0.25$ ) and find that such alignments (see right panel of Fig. 3) are present also in fainter red-sequence galaxies as well as in the orientation of groups of galaxies in the extended environment around the cluster (see also Kitzbichler \& Saurer 2003).

\subsection{Group shape-richness connection}

Plionis, Basilakos \& Tovmassian (2004) and Plionis \& Basilakos (2004) analysed two large samples of groups (the UzC-SsRs2 and the 2DFGRS-2PIGG samples) within a roughly volume-limited region ( $c z \leqslant 5500$ and $c z \leqslant 30000 \mathrm{~km} / \mathrm{sec}$ for the two samples respectively). In Fig. 4 we present the projected, on the plane of the sky, axial ratio distribution for three group richness classes, while in Fig. 5 we show the corresponding inverted axial ratio distribution assuming that groups are intrinsically either prolate or oblate spheroids.

There is an obvious group richness-flatness relation, seen in both projected and intrinsic axial ratio distributions, with group flatness decreasing with richness. We also find a weak (Spearman correlation coefficient $\mathcal{R}=-0.13)$ but significant correlation $\left(\mathcal{P}<10^{-6}\right)$, between the velocity dispersion of groups and their flattening, with flatter systems having lower velocity dispersions. Such a correlation as well as the increase of the group sphericity 


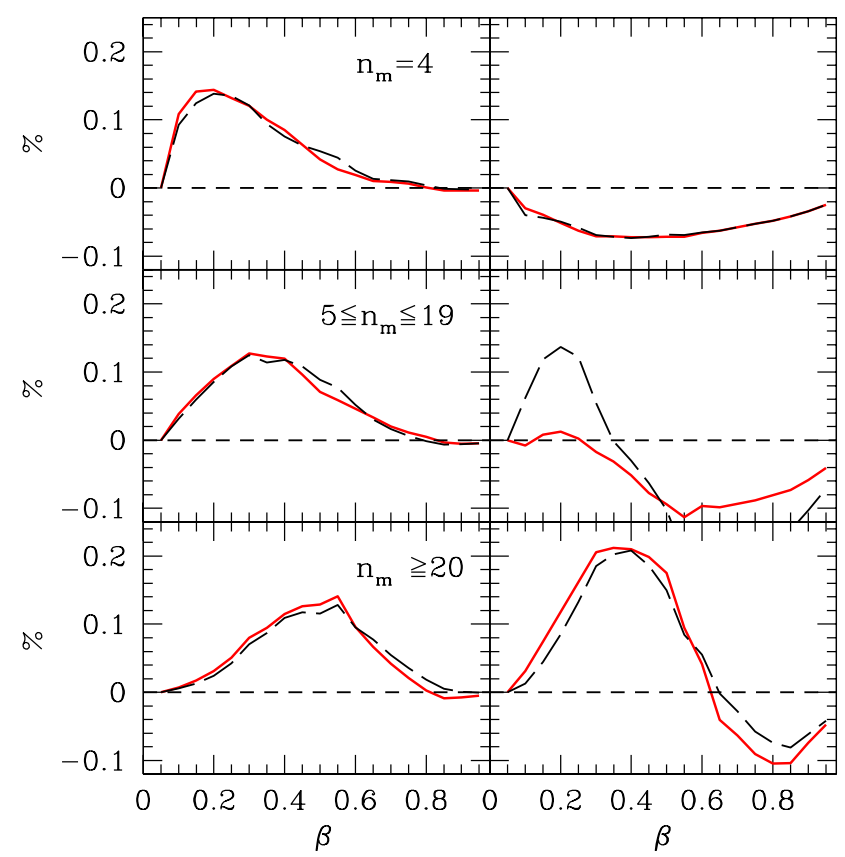

Figure 5. RIGHT PANEL: The intrinsic distribution of group axial ratios for the NGP (continuous line) and SGP (dashed line) subsamples assuming that they are either prolate (left panel) or oblate (right panel) spheroids. The unphysical negative values of the latter model indicates its failure to represent the true three-dimensional shape of groups.

with richness, which extends also to clusters, could be explained as an indication of a higher degree of virialization, which is expected to be more rapid in more massive systems. Furthermore, we find that groups of galaxies are prolate-like systems which is in agreement with the shape of clusters and superclusters of galaxies (e.g. Cooray 2000; Basilakos, Plionis \& Maddox 2000 and references therein). This is the expected shape for cosmic structures that form in quasi one-dimensional large-scale structures (filaments). Other interesting environmental effects that have been found is the decrease of the group velocity dispersion as their distance from nearby clusters increases (e.g. Ragone et al 2004).

\section{Conclusions}

We have presented evidence, based on the APM and Abell cluster samples as well as the UzC-SssRS2 and 2DFGRS group samples, that there is a strong link between the dynamical state of clusters/groups of galaxies and their large-scale environment. Dynamically young clusters are significantly more aligned with their nearest neighbors and they are also much more spatially clustered. Clusters belonging to dense superclusters are preferentially aligned with their parent supercluster projected orientation; bright galaxies, in dynamically young clusters, are also orientated along their parent cluster major axis. These coherent orientation effects, from the scale of galaxies to that of superclusters, support the hierarchical formation scenario in which clusters form by anisotropic merging along the large-scale filamentary structures within which they are embedded. 


\section{Acknowledgements}

This work has been partly funded within the framework of the program 'Promotion of Excellence in Technological Development and Research', project 'X-ray Astrophysics with ESA's mission XMM', as well as by the Mexican Government grant No CONACyT2002-C01-39679.

\section{References}

Bingelli, B., 1982, A\&A, 250, 432

Bekki, K. 1982, ApJ, 510, L15

Bond, J.R. 1986 Galaxy Distances and Deviations from the Hubble Flow, eds. Madore, B.F., Tully, R.B., (Dordrecht: Reidel), 255

Buote, D. \& Tsai, J. 1996, ApJ, 458, 27

Cooray, R.A., 2000, MNRAS, 313, 783

Eke, V.K., et al., 2004, MNRAS, 348, 866

Evrard A.E., Mohr J.J., Fabricant D.G., Geller M.J.,1993, ApJ, 419, L9

Faltenbacher, A., Gottlöber, S., Kerscher, M., Müller, V., 2002, A\&A, 395, 1

Floor, S.N., Melott, A.L., Miller, C.J., Bryan, G.L., 2003, ApJ, 591, 741

Jeltema, T.E., Canizares, C.R., Bautz, M.W., Buote, D.A., 2004, in "Multiwavelength Cosmology" proceedings, Ed. M.Plionis, Kluwer Academic Publishers

Knebe, A., Gill, S.P.D., Gibson, B.K., Lewis, G.F., Ibata, R.A., Dopita, M.M., 2004, ApJ, in press, astro-ph/0311202

Kitzbichler, M.G. \& Saurer, W., 2003, ApJ, 590, L9

Kolokotronis, V., Basilakos, S., Plionis, M., Georgantopoulos, I., 2001, MNRAS, 320, 49

Lacey, C., Cole, S., 1996, MNRAS, 262, 627

Loken, C., Melott, A.L., Miller, C.J., 1999, ApJ, 520, L5

Mohr, J.J., Evrard, A.E., Fabricant, D.G., Geller, M.J., 1995, ApJ, 447, 8

Onuora, L.I., Thomas, P.A., 2000, MNRAS, 319, 614

Plionis M., 1994, ApJS, 95, 401

Plionis M., 2001, in Clusters and the High-Redshift Universe observed in X-rays XXI ${ }^{\text {th }}$ Moriond Astrophysics Meeting, eds. Neumann et al., in press

Plionis M. \& Basilakos, S., 2002, MNRAS, 329, L47

Plionis, M., Benoist, C., Maurogordato, S., Ferrari, C. \& Basilakos, S., 2003, ApJ, 594, 144

Plionis, M., Basilakos, S. \& Tovamssian, H., 2004, MNRAS, in press

Plionis, M., Basilakos, S., 2004, in preparation

Richstone, D., Loeb, A., Turner, E.L., 1992, ApJ, 393, 477

Ragone, CJ., Merchán, M., Muriel, H. \& Zandivarez, A., 2004, astro-ph/0402155

Ramella, M., Geller, M. J., Pisani, A., \& da Costa, L., 2002, AJ, 2976, 123

Rizza E., Burns J. O., Ledlow M. J., Owen F. N., Voges, W., Blito M., 1998, MNRAS, 301, 328

Sarazin, C.L., 2001, in Merging Processes in clusters of Galaxies, eds. Feretti, L., Gioia, M., Giovannini, G., (Dordrecht: Kluwer).

Schindler S., 1999, Vulcano Workshop 1999, Multifrequency Behaviour of High Energy Cosmic Sources, eds. Giovanelli F., Sabau-Graziati L.

Schuecker P., Böhringer H., Reiprich T. H., Feretti L., 2001, A\&A, 378, 408

Splinter, R.J., Melott, A.L., Linn, A.M., Buck, C., Tinker, J., 1997, ApJ, 479, 632

Struble, M.F., Peebles, P.J.E., 1985, AJ, 90, 582

van Haarlem, M., van de Weygaert, R., 1993, ApJ, 418, 544

Wang, Q.D. \& Ulmer, M.P., 1997, MNRAS, 292, 920

West, M.J., Villumsen, J.V., Dekel, A., 1991, ApJ, 369, 287

West, M.J., 1994, MNRAS, 268, 79

West, M.J., Jones C. \& Forman W., 1995, ApJ, 451, L5

West, M.J. \& Blakeslee, J.P., 2000, ApJ, 543, L27 